\title{
Conditioned umbilical cord tissue provides a natural three-dimensional storage compartment as in vitro stem cell niche for human mesenchymal stroma/stem cells
}

\author{
Yuanyuan Yang ${ }^{1,2}$, Catharina Melzer ${ }^{1}$, Vesna Bucan ${ }^{3}$, Juliane von der Ohe ${ }^{1}$, Anna Otte ${ }^{1}$ and Ralf Hass ${ }^{1,4^{*}}$
}

\begin{abstract}
Background: The use of large amounts of human multipotent mesenchymal stroma/stem cells (MSC) for cell therapies represents a desirable property in tissue engineering and banking in the field of regenerative medicine.

Methods and results: Whereas cryo-storage of umbilical cord (UC) tissue pieces in liquid nitrogen without ingredients was associated with predominant appearance of apoptotic cells after thawing and re-culture, progressive growth of MSC was observed following use of cryo-medium. Moreover, conditioning of UC tissue pieces by initial explant culture and subsequent cryo-storage with cryo-medium accelerated a further MSC culture after thawing. These findings suggested that conditioning of UC tissue pieces provides an in vitro stem cell niche by maintenance of a 3-dimensional natural microenvironment for continuous MSC outgrowth and expansion. Indeed, culture of GFP-labeled UC tissue pieces was accompanied by increased outgrowth of GFP-labeled cells which was accelerated in conditioned UC tissue after cryo-storage. Moreover, cryopreserved conditioned UC tissue pieces in cryo-medium after thawing and explant culture could be cryopreserved again demonstrating renewed MSC outgrowth after repeated thawing with similar population doublings compared to the initial explant culture. Flow cytometry analysis of outgrowing cells revealed expression of the typical MSC markers CD73, CD90, and CD105. Furthermore, these cells demonstrated little if any senescence and cultures revealed stem cell-like characteristics by differentiation along the adipogenic, chondrogenic and osteogenic lineages.
\end{abstract}

Conclusions: Expression of MSC markers was maintained for at least 10 freeze/thaw/explant culture cycles demonstrating that repeated cryopreservation of conditioned UC tissue pieces provided a reproducible and enriched stem cell source.

Keywords: Mesenchymal stroma/stem cells, Tissue conditioning, Umbilical cord tissue, Cryopreservation, Tissue engineering, Three-dimensional long-term culture

\section{Background}

Mesenchymal stroma/stem cells (MSC) are recruited towards tissue injuries to exhibit repair, immune modulation, neovascularization, and differentiation capacity for support of tissue regeneration. Transplantation of

\footnotetext{
* Correspondence: hass.ralf@mh-hannover.de

'Department of Obstetrics and Gynecology, Biochemistry and Tumor Biology Laboratory, Hannover Medical School, Carl-Neuberg-Str. 1, Hannover D-30625, Germany

${ }^{4}$ Department of Gynecology and Obstetrics, Biochemistry and Tumor Biology Laboratory, Hannover Medical School, Carl-Neuberg-Straße 1, Hannover D 30625, Germany

Full list of author information is available at the end of the article
}

MSCs for substitution of damaged cells or tissue as well as MSC expansion for tissue engineering in the field of regenerative medicine require appropriate cell sources which are provided by invasive collection of bone marrow-derived MSC [1] or adipose tissue-derived MSC [2] from appropriate biopsies. Alternatively, more easily accessible, noninvasive, and therefore ethically noncontroversial MSC sources are represented by neonatal tissues, including MSC from placenta or from umbilical cord (UC) [3, 4]. Previous studies revealed the advantages of explant cultures from human UC to 
obtain large quantities of human UC-MSC with longterm maintenance of stem cell properties [5].

Human MSC are characterized as a multipotent heterogeneous cell population which can be found in nearly all vascularized organs and tissues. The stem cell properties include self-renewal and regenerative potential [6]. Certain heterogeneity both in morphology and cell fate as demonstrated by isolation of MSC subpopulations [7] may result partially from interaction with neighboring cells in the cellular microenvironment, from altered trophic factors, $\mathrm{pH}$, hypoxic conditions, or oxidative stress and provide difficulties in MSC characterization. Additional heterogeneous and even controversial findings provided a discussion platform for a clear description and definition of MSC [8]. Consequently, instead of a certain specific cell marker, a broad spectrum of simultaneous minimal criteria has been identified for MSC, including the capability for plastic adherence, paralleled by expression of the CD73, CD90, and CD105 surface molecules with concomitant absence of other cell type-specific markers such as CD14, CD31, CD34, CD45, and human leukocyte antigen DR (HLA-DR) [9, 10]. Furthermore, MSC exhibit migratory activities [11] and display at least a tri-lineage differentiation capacity along the osteogenic, chondrogenic, and adipogenic phenotype [9] whereby also transgermline maturation pathways have been discussed. The differentiation potential of MSC together with immunemodulatory functions and an involvement in neovascularization represent multipotent cellular functionalities which are required for the use in regenerative medicine. MSC have thus been successfully applied in a variety of clinical trials - for example, large bone defects [12], cartilage lesions and traumatic articular cartilage defects [13], osteogenesis imperfecta [14], graft versus host disease [15], spinal cord injuries [16], cardiovascular diseases [17], and hematological pathologies [18] - indicating the need for large quantities of MSC.

A reproducible source for healthy stem cells including MSC could be represented by appropriately cryopreserved tissue. Previous work has demonstrated that cryopreservation of birth-associated tissues such as placenta and UC can serve for hematopoietic stem cells and MSC, respectively [19, 20]. Moreover, MSC can also be obtained after cryostorage of whole adipose tissue [21]. Several other studies using different fertility preservation strategies included embryo cryopreservation, oocyte vitrification, and ovarian tissue cryostorage [22]. In addition, counteracting certain mechanisms of post-thaw cell death could increase the quality of cryopreserved tissue [23]. In the present work, we demonstrate that conditioning of UC tissue pieces can minimize post-thaw cell death and serves as a reproducible MSC source and as a valuable three-dimensional storage compartment for repeatable cryopreservation and maintenance of MSC within their natural microenvironment.

\section{Materials and methods \\ Cell and tissue culture of human UC and MSCs}

The use of primary human MSC following explant culture from UC tissue was approved by the Ethics Committee of Hannover Medical School (Project \#443) on 26 February 2009. Informed written consent was obtained from each patient.

MSC-like cells were isolated from human UCs as reported previously [5]. The cells were obtained from UC explant cultures of three different patients following delivery of full-term (38-40 weeks) infants either spontaneously or by Cesarean section. In brief, UC tissue was washed with phosphate buffer saline (PBS) to remove blood cells, cut into approximately $0.5 \mathrm{~cm}^{3}$ large pieces, and incubated in alpha-minimum essential medium ( $\alpha M E M$; Sigma Chemie GmbH, Steinheim, Germany) supplemented with $15 \%$ allogeneic human AB-serum (HS; commercially obtained from blood bank, University Campus Lübeck, Germany), $100 \mathrm{U} / \mathrm{ml}$ penicillin, $100 \mu \mathrm{g} /$ $\mathrm{ml}$ streptomycin, and $2 \mathrm{mM} \mathrm{L}$-glutamine (Sigma) at $37^{\circ} \mathrm{C}$ in a humidified atmosphere with $5 \%$ carbon dioxide. After about 14 days of explant culture with fresh medium replacement every 3-4 days, the UC tissue pieces were removed and the adherent cells were harvested by accutase (Sigma) treatment for 3 minutes at $37^{\circ} \mathrm{C}$. The obtained cell suspension was centrifuged at $320 \times g$ for 5 minutes and the cells were resuspended in MSC culture medium ( $\alpha$ MEM supplemented with $10 \% \mathrm{HS}, 100 \mathrm{U} / \mathrm{ml}$ penicillin, $100 \mu \mathrm{g} / \mathrm{ml}$ streptomycin, and $2 \mathrm{mM}$ L-glutamine) and subcultured in the appropriate passage.

The UC tissue pieces after initial explant culture were termed "conditioned" UC tissue. Conditioned tissue has been cultured for approximately 2 weeks allowing adaptation to the culture conditions in contrast to freshly prepared tissue. Cryopreservation of UC tissue was performed in cryomedium (90 \% HS containing $10 \%(\mathrm{v} / \mathrm{v})$ dimethyl sulfoxide (DMSO)) with a freezing velocity of approximately $1{ }^{\circ} \mathrm{C} /$ minute (Nalgene Cryo $1{ }^{\circ} \mathrm{C}$ freezing container; Nunc: Wiesbaden, Germany) until the samples reached $-80{ }^{\circ} \mathrm{C}$. Thereafter, the cryopreserved UC tissues were stored in liquid nitrogen for 3 days until start of the next explant culture.

Green fluorescent protein (GFP) labeling of UC tissue pieces was performed by lentiviral transduction. Six UC tissue pieces of similar size were transduced with a third-generation lentiviral SIN vector containing the eGFP gene according to a labeling technique described previously for the transduction of MSCs [24]. Briefly, each of the six UC tissue pieces was separately centrifuged together with the lentivirus at $2000 \times g / 30$ minutes, washed three times in MSC culture medium, 
and incubated in one well of a 24-well plate with $500 \mu \mathrm{l}$ MSC medium. Fluorescence was measured at $480 \mathrm{~nm}$ excitation/520 nm emission wavelength using a Fluoroskan Ascent FL (Thermo Scientific, Waltham, MA, USA) after transfer of the labeled UC tissue pieces into one well of a 96-well microtiter plate, respectively. Following fluorescence measurement, the labeled UC tissue pieces were cultured further in the 24-well plates.

\section{Reverse transcription PCR analysis}

Total RNA was extracted using the RNeasy Mini Kit (Qiagen, Hilden, Germany) according to the manufacturer's instructions and reverse transcription RT-PCR was performed as described previously [25]. As a template, 2.5 $\mu \mathrm{l}$ cDNA was used with primers specific for CD73 (sense, 5'-CGC AAC AAT GGC ACA ATT AC-3'; antisense, 5'-CTC GAC ACT TGG TGC AAA GA-3'; amplification product 241 base pairs (bp)), CD90 (sense, 5'-GGA CTG AGA TCC CAG AAC CA-3'; antisense, 5'-ACG AAG GCT CTG GTC CAC TA-3'; amplification product 124 bp), CD105 (sense, 5'-TGT CTC ACT TCA TGC CTC CAG CT-3'; antisense, 5'-AGG CTG TCC ATG TTG AGG CAG T-3'; amplification product $378 \mathrm{bp}$ ), and eGFP (sense, 5'-CTA TAT CAT GGC CGA CAA GCA GA-3'; antisense, 5'-GGA CTG GGT GCT CAG GTA GTG G-3'; amplification product $165 \mathrm{bp}$ ). As a control, glyceraldehyde 3-phosphate dehydrogenase (GAPDH) PCR (sense, 5'-ACC ACA GTC CAT GCC ATC AC-3'; antisense, 5'-TCC ACC ACC CTG TTG CTG TA-3'; amplification product $452 \mathrm{bp}$ ) was performed (all primers customized by Eurofins, MWG GmbH, Ebersberg, Germany). Aliquots of $25 \mu \mathrm{l}$ of each RT-PCR product were separated on a $2 \%$ agarose gel including the standard GeneRuler 100 bp DNA Ladder (Thermo Scientific) and visualized by GelRed ${ }^{\mathrm{m} x}$ (Biotium Inc., Hayward, CA, USA) staining.

\section{Cell cycle analysis}

The cell cycle analysis in the different MSC populations was performed as described previously [26]. Briefly, $5 \times$ $10^{5}$ cells were fixed in $70 \%(\mathrm{v} / \mathrm{v})$ ice-cold ethanol at $4{ }^{\circ} \mathrm{C}$ for 24 hours. The fixed cells were stained with the CyStain DNA 2 step kit (Partec GmbH, Münster, Germany) and filtered through a $50 \mu \mathrm{m}$ filter. Thereafter, samples were analyzed in a Galaxy flow cytometer (Partec) using the FloMax analysis software (Partec).

\section{Senescence-associated $\beta$-galactosidase assay}

Cell senescence can be detected by different expression levels of senescence-associated $\beta$-galactosidase (SA- $\beta$-gal) [27]. The amount of senescent cells was determined using the SA- $\beta$-gal staining kit (Cell Signaling Technology, Danvers, Massachusetts, USA) and 4',6-diamidine-2'phenylindoldihydrochloride (DAPI; Roche Diagnostics
$\mathrm{GmbH}$, Mannheim, Germany) fluorescence counterstain in accordance with the manufacturer's instructions. For this purpose, about $4000 \mathrm{cells} / \mathrm{cm}^{2}$ were cultivated for 48 hours in the corresponding media before fixation and SA$\beta$-gal staining. After completion of the staining procedure, four representative images were taken from diverse areas of each cell culture using phase-contrast microscopy and $\mathrm{Cell}^{\mathrm{B}}$ Imaging Software (Olympus GmbH, Hamburg, Germany). For the calculation of the percentage of senescent cells, the total number of cell nuclei and the number of cell nuclei surrounded by cyan dye were enumerated.

\section{Analysis of surface markers by flow cytometry}

Characterization of the MSC immunophenotype was performed as described previously [7]. Continuously proliferating MSC were harvested and analyzed for cell surface marker expression by flow cytometry. After blocking nonspecific binding to Fc-receptors by incubation of $10^{6}$ cells with $2 \%$ fetal calf serum (FCS) for 15 minutes at $4{ }^{\circ} \mathrm{C}$ and washing with PBS-bovine serum albumin (BSA), the cells were incubated with the following appropriately-labeled monoclonal anti-human antibodies: CD73-phycoerythrin (PE) (clone AD2; BD Bioscience, Heidelberg, Germany), CD90-PE (clone 5E10, IgG1; BioLegend Inc., San Diego, CA, USA), CD105-PE (clone 43A3, IgG1; BioLegend Inc.), CD14-fluorescein isothiocyanate (FITC) (clone TÜK4, IgG2a; MACS, Miltenyi, Bergisch-Gladbach, Germany), CD31-FITC (clone WM59, IgG1; DAKO, Hamburg, Germany), CD34-PE (clone AC136, IgG2a; DAKO), and CD45-FITC (clone T29/33, IgG1; DAKO). Following antibody staining, all samples were washed twice with PBSBSA. Positive staining was obtained according to control measurements of the different populations with isotypematching IgG control antibodies. Flow cytometry analysis and histograms were performed in a Galaxy FACSan (Partec) using FloMax analysis software (Partec).

\section{Cell differentiation}

Osteogenesis was induced by maintaining confluent cell cultures in Dulbecco's modified Eagle's medium (DMEM: Dulbecco's Modified Eagles's Medium)/F12 supplemented with $0.15 \mathrm{mM}$ ascorbat-2-phosphate, $0.1 \mu \mathrm{M}$ dexamethasone, $3 \mathrm{mM} \beta$-glycerol, $10 \%$ fetal bovine serum (FBS; Biochrom, Berlin, Germany), and $1 \%$ penicillin-streptomycin for 4 weeks. Differentiation was visualized by light microscopy and Alizarin red staining.

For adipogenic differentiation the different MSC cultures were first grown to confluence and then cultured in the appropriate differentiation medium (DMEM/F12 with L-glutamine (PAA, Cölbe, Germany) with $10 \%$ FBS, $1 \%$ penicillin-streptomycin, $0.15 \mathrm{mM}$ ascorbat-2-phosphate, $1 \mu \mathrm{M}$ dexamethasone, $0.5 \mathrm{mM}$ 3-isobutyl-1-methylxanthine, $0.1 \mathrm{mM}$ indomethacin, and $4 \mathrm{U} / \mathrm{l}$ insulin (Aventis, Frankfurt, Germany)) for 14 days, respectively. The 
maturity of an adipocyte phenotype was evaluated by light microscopy and oil red $\mathrm{O}$ staining.

For chondrogenic differentiation, cells were kept in pellet culture after centrifugation at $300 \times g$ for $5 \mathrm{mi}-$ nutes. The cultures were cultivated in DMEM/F12 supplemented with $0.15 \mathrm{mM}$ ascorbat-2-phosphate, $1 \%$ insulin, transferrin, selenium, ethanolamine solution (ITS-X; Life Technologies, Darmstadt, Germany), 100 $\mathrm{mM}$ sodium pyruvate (Biochrom), $0.1 \mu \mathrm{M}$ dexamethasone, $0.35 \mathrm{mM}$ proline, and $10 \mathrm{ng} / \mathrm{ml}$ TGF $\beta 1$ (Peprotec, Rocky Hill, NJ, USA) for 3 weeks. Afterwards, the pellets were rinsed twice in PBS and fixed in $4 \%$ formaldehyde in PBS, embedded in paraffin, and cut into sections of 5 $\mu \mathrm{m}$ thickness. The sections were stained with alcian blue for detection of glycosaminoglycans.

\section{Results}

Direct cryopreservation of freshly prepared UC tissue pieces in liquid nitrogen without cryomedium and a following reculture in MSC medium was associated with the production of viscous material in the supernatant and appearance of debris and dead cells within 14 days (Fig. 1a, upper panel). Supportive evidence was obtained by cell cycle analysis of this culture demonstrating predominantly DNA fragments in the sub-G1 phase as an indication for cell death (Fig. 1b, upper panel). In contrast, reculture of UC tissue pieces previously cryopreserved in the presence of cryomedium revealed the initial production of viscous material and the outgrowth of MSC-like cells after 14 days (Fig. 1a, bottom panel), which was paralleled by a cell cycle of a proliferating population demonstrating cells in G0/G1, S, and G2/M phases (Fig. 1b, bottom panel).

Alternatively, direct explant culture of freshly prepared UC tissue for about 20 days was accompanied by initial outgrowth of MSC-like cells, whereby the UC tissue became "conditioned". Liquid nitrogen cryopreservation of these conditioned UC tissue pieces with cryomedium followed by reculture exhibited an outgrowth of viable MSC-like cell populations already within 8 days (Fig. 1c, upper panel), whereby the first cells were observed within 2 days of reculture. These differences demonstrated that the outgrowth of cells from the conditioned UC tissues starts immediately after reculture, in contrast to freshly prepared UC tissue still requiring adaptation to the culture conditions. Moreover, a second cryopreservation of these recultured conditioned UC100314 tissue pieces in liquid nitrogen and another second reculture (UC100314-Re) was accompanied by a similar outgrowth of MSC-like cells within 14 days (Fig. 1c, bottom panel).

These findings underscored the use of cryomedium for tissue preservation and suggested that the process of UC tissue conditioning involved an intratissue remodeling to enable growth stimulation with protection of the new cells. Histochemical analysis after hematoxylin and eosin staining of UC tissue confirmed morphological changes by a marked condensation of the vessels and the connective tissue within the UC after 14 days of conditioning and in recultured UC tissue after cryopreservation as compared with fresh UC tissue (Fig. 2a).

The expression of MSC characteristic genes was also observed in the UC tissue pieces under the different conditions. RNA from fresh UC tissue as well as 14-day conditioned UC tissue or conditioned UC tissue after cryopreservation and reculture demonstrated transcripts for CD73, CD90, and CD105 (Fig. 2b).

The conditioning of UC tissue was investigated in six UC tissue pieces of approximately similar size and weight which were cultured for up to 19 days with prior GFP labeling of tissue-associated cells via lentiviral transduction. During this explant culture, progressively increasing GFP fluorescence intensities of the tissue pieces by $3.4 \pm 1.7$-fold $(n=6 ; p<0.05)$ were measured (Fig. 2c). Previous work has demonstrated that the GFP fluorescence intensities corresponded to an appropriate cell number [24]. Consequently, conditioning of UC tissue was associated with a significant increase of proliferating cells within the tissue. To further address this hypothesis, six conditioned UC tissue pieces after cryopreservation were recultured and similarly GFP labeled. The conditioned tissue pieces were more round-shaped (Fig. 2d) compared with the initial freshly prepared tissue as observed previously [5]. Already within 7 days of explant culture the fluorescence intensity of the conditioned tissue pieces increased by $11.0 \pm 5.1$-fold $(n=6: p$ $<0.005)$, which substantiated enhanced and accelerated cell growth after tissue conditioning (Fig. 2d) as compared with the initial fresh culture that took about 19 days with a much lower fluorescence intensity (Fig. 2c).

Following 21 days of explant culture, quantification and phenotyping of the outgrowing cells from $5.2 \mathrm{mg}$ GFP-transduced UC tissue were performed by flow cytometry analysis. A total yield of $1.2 \times 10^{5}$ cells contained about $6 \%$ of GFP-positive cells (Fig. 3a, left panel). Flow cytometry evaluation of the MSC-typical markers CD73, CD90, and CD105 always revealed in total more than $98 \%$ positive cells, whereby about $93-$ $94 \%$ exhibited a PE signal of the unlabeled cells. The remaining about 5-6 \% reflected the outgrowing cells with a double fluorescence of PE and GFP (detected by the FITC channel) in accordance with the transduced population (Fig. 3a, right panel).

A direct comparison between fresh and conditioned UC tissue substantiated an advantage of the conditioning whereby all outgrowing cells from both tissues expressed the MSC-typical markers CD73, CD90, and CD105 (Fig. 3b). A 13-day explant culture revealed about $1.2 \times$ $10^{3}$ cells/mg fresh UC tissue (Fig. 3b, left panel) whereas 

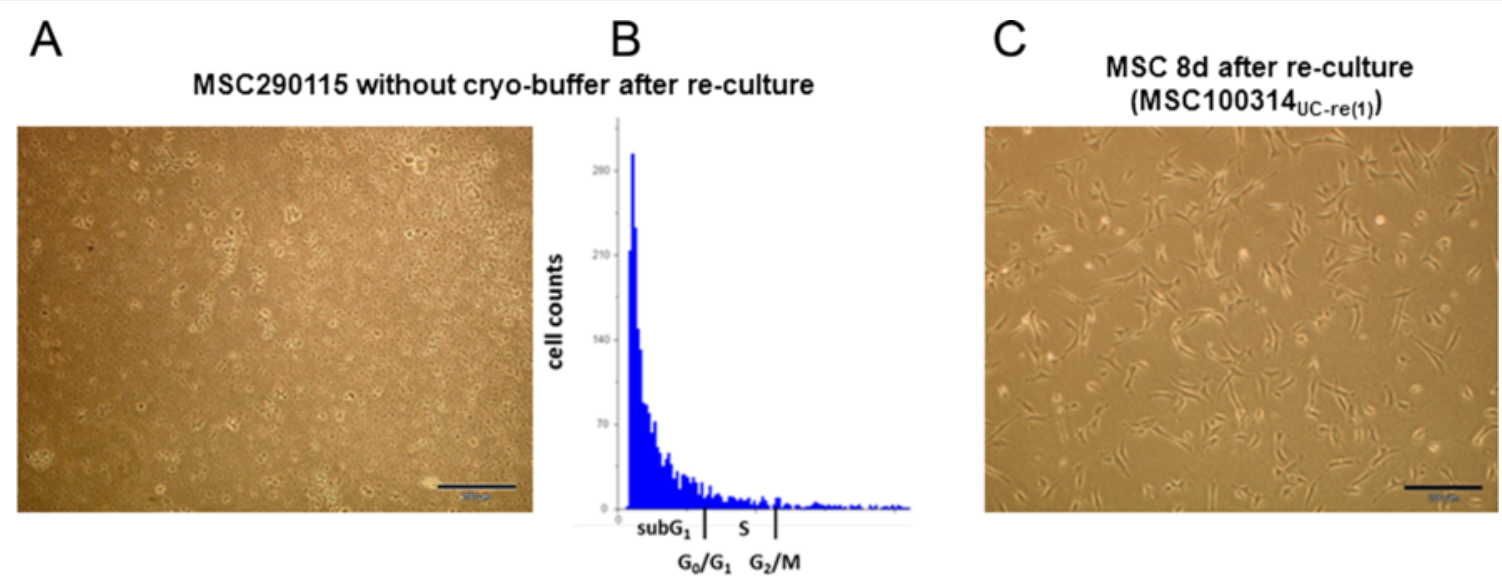

MSC290115 with cryo-buffer after re-culture
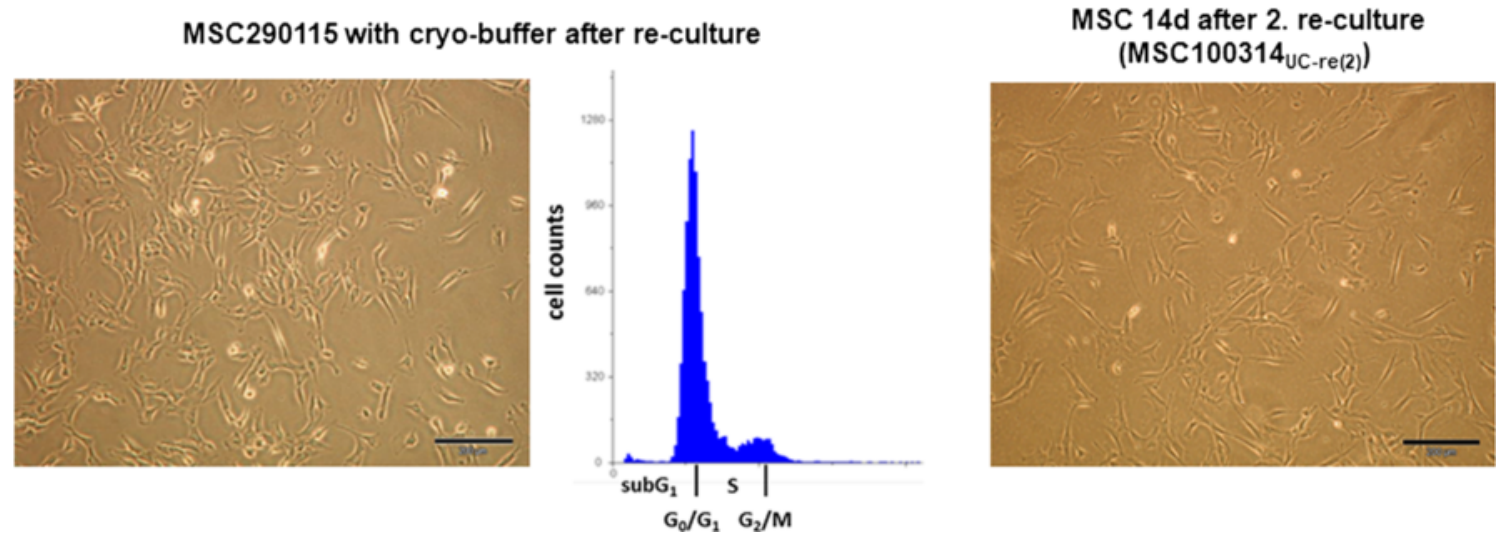

Fig. 1 Morphology and cell cycle properties of recultured UC tissue. a Cryopreserved pure UC290115 tissue pieces in liquid nitrogen without cryobuffer or any other additives (upper panel) and in the presence of cryobuffer (lower panel) were recultured in MSC medium for 14 days and the morphology of potential MSC was documented (magnification bars $=100 \mu \mathrm{m}$ ). b Potential MSC from 14-day explant cultures of recultured and previously cryopreserved UC290115 tissue pieces in liquid nitrogen without cryobuffer (upper panel) and in the presence of cryobuffer (lower panel) were harvested and subjected to cell cycle analysis. Distribution within sub-G1, G0/G1, S, and G2/M cell cycle phases was performed by FloMax cell cycle software. c Reculture of conditioned UC100314re(1) tissue pieces after cryopreservation in liquid nitrogen with cryobuffer revealed the outgrowth of MSC-like cells (MSC100314 $\mathrm{UC}_{\text {-re(1) }}$ ) within 8 days (upper panel). In addition, a second reculture after repeated cryopreservation of these UC tissue pieces (UC100314 re(2) ) demonstrated an explant culture of MSC-like cells (MSC100314 UC-re(2)) within 14 days (magnification bars $=100 \mu \mathrm{m})$. MSC mesenchymal stroma/stem cells

prior conditioning yielded about $5.8 \times 10^{4}$ cells/mg conditioned tissue (Fig. 3b, right panel).

Together, these findings demonstrated that cells including GFP-labeled populations are proliferating within the UC tissue during conditioning. Consequently, conditioned UC tissue represented an accelerated initial outgrowth during explant culture whereby explanted cells exhibit MSC-like properties.

Cells obtained after reculture of conditioned and cryopreserved UC tissues from three individual donors were further analyzed for stem cell characteristics including proliferative capacity, expression of MSC markers, and differentiation potential along mesenchymal lineages. The three primary cultures MSC100314 $4_{\text {UC-re(1) }}$, MSC110314 $4_{\text {UC-re(1) }}$, and MSC180314 $4_{\text {UC-re(1) }}$, obtained from corresponding cryopreserved and recultured UC tissues, respectively, exhibited significant proliferative activity (Fig. 4a). Moreover, cells from the second reculture after repeated cryopreservation of the same tissues (MSC100314 $4_{\mathrm{UC}-\mathrm{re}(2)}$,

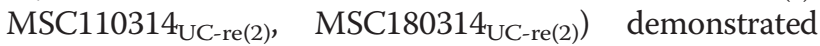
similar cell growth potential compared with the explant cultures from the first UC reculture (Fig. 4a). Likewise, all six populations revealed continuous cell cycle progression and little if any differences were detectable in the pattern and distribution of cell cycle phases between cell populations from the first or the second reculture of UC tissues (Fig. 4b). These data pointed to a constant growth capacity of cells irrespective of one or two freezing cycles of the originating conditioned tissue. Further support was obtained by the measurement of SA- $\beta$-gal, with less than $2 \%$ of positive cells in all cultures indicating that even after the second 


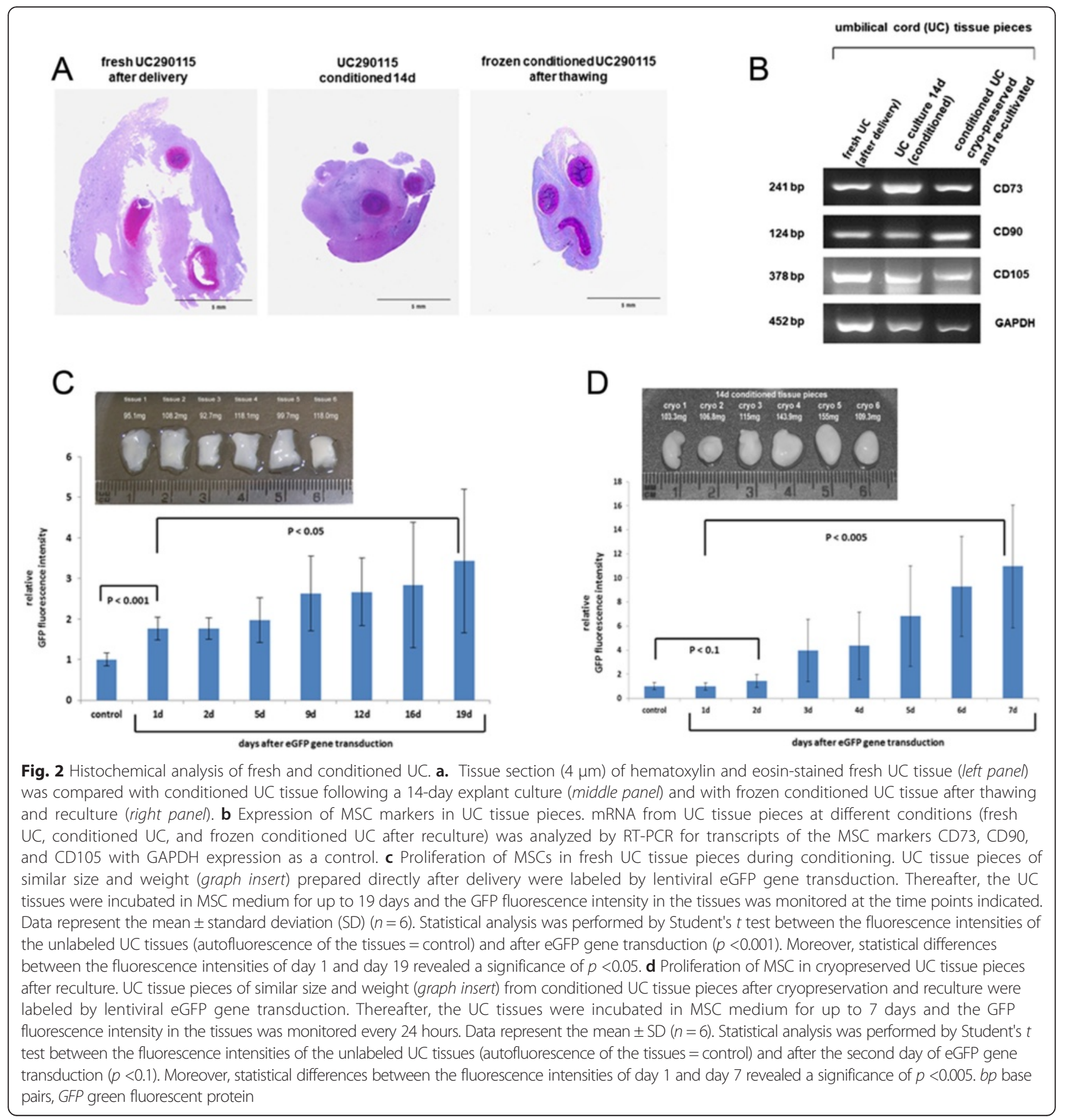

reculture of cryopreserved conditioned UC tissue a majority of outgrowing juvenile and proliferative active cells prevailed (Fig. 4c).

MSC characteristics in these explant populations were evaluated by the presence of CD73, CD90, and CD105, respectively. Flow cytometry analysis demonstrated always more than $90 \%$ of these MSC-associated surface markers in MSC100314 $4_{\mathrm{UC}-\mathrm{re}(1)}, \mathrm{MSC}_{110314_{\mathrm{UC}-\mathrm{re}(1)} \text {, and }}$ MSC180314 ${ }_{\text {UC-re(1) }}$, as well as in corresponding cell populations derived from reculture of the second cryopreservation (MSC100314 $4_{\text {UC-re(2), }}$ MSC110314 $4_{\text {UC-re(2), }}$, MSC180314 ${ }_{\mathrm{UC}-\mathrm{re}(2)}$ ) (Fig. 5), respectively. These findings were paralleled by little if any detectable CD14, CD31, CD34, or CD45 surface markers in either population (Fig. 5), further substantiating the outgrowth of MSClike cells in the explant cultures.

Stem cell properties of MSC also include the capability to differentiate at least along the osteogenic, adipogenic, 


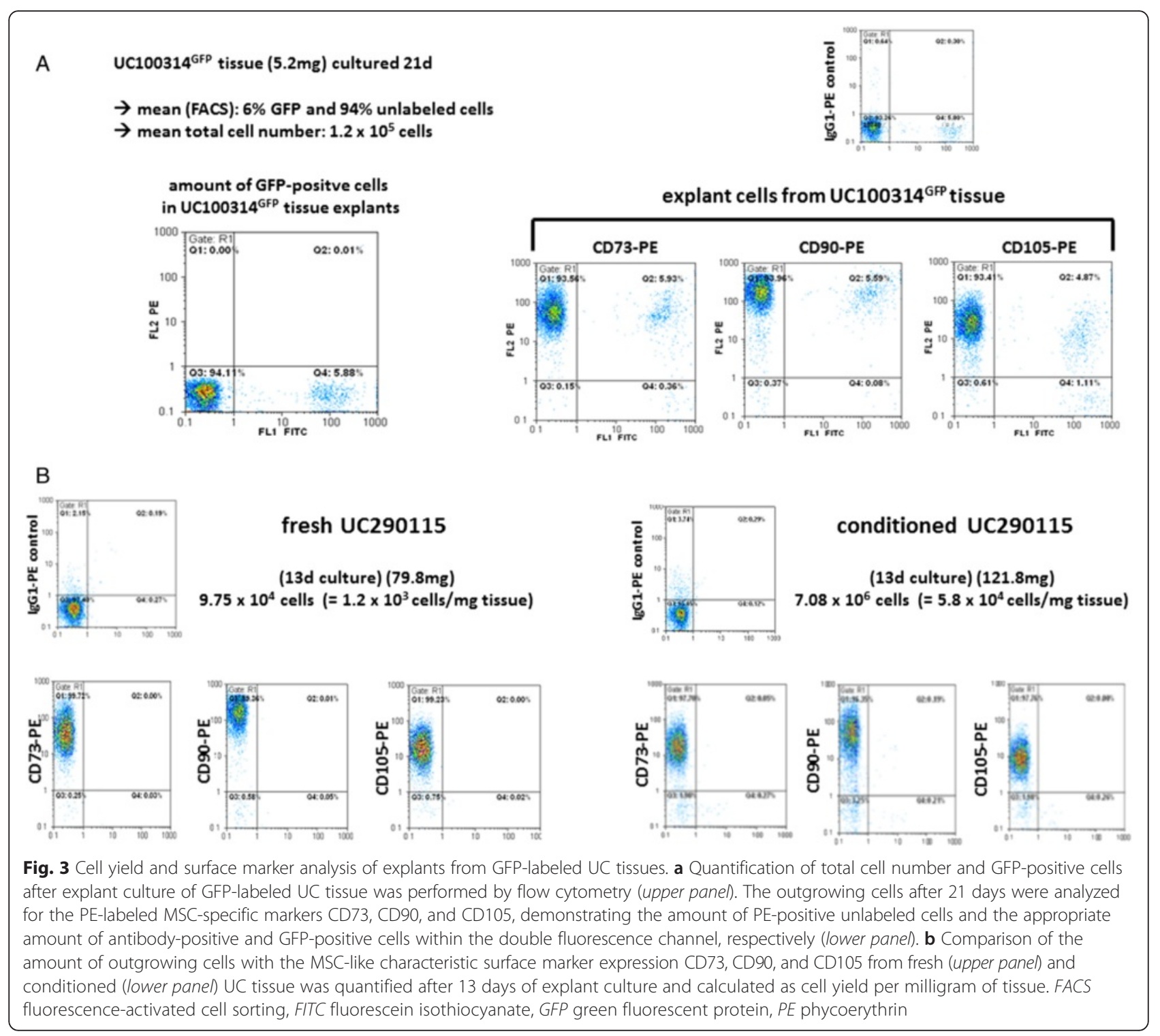

and chondrogenic lineage. Induction of this tri-lineage maturation revealed strong similarities in the differentiation potential of the MSC100314 $4_{\text {UC-re(1) }}$, MSC110314 $_{\text {UC-re(1), }}$ and MSC180314 $4_{\text {UC-re(1) }}$ explant cultures and from the second reculture after repeated cryopreservation (MSC100314 $4_{\mathrm{UC}-\mathrm{re}(2)}$, (Fig. 6). Thus, mineralization of the explant cultures assayed by Alizarin red staining demonstrated osteogenic potency in contrast to undifferentiated controls (Fig. 6, left panels). Moreover, adipogenic potential is usually demonstrated by increased lipid metabolism associated with the formation of intracellular lipid droplets stained with oil red $\mathrm{O}$ which could be observed exclusively in the adipogenic differentiation-induced explant populations (Fig. 6, middle panels). A variety of sulfated proteoglycan deposits are properties of functional chondrocytes which can be determined by alcian blue, and significantly enhanced staining was detected in the differentiated cultures compared with the undifferentiated controls (Fig. 6, right panels).

Maintenance of MSC stemness by repeated freeze/ thaw/explant culture cycles of UC tissue revealed an unaltered cell morphology (data not shown). Moreover, all cultures demonstrated consecutive expression of the MSC-specific markers CD73, CD90, and CD105 starting from the first explant culture during conditioning of MSC tissue until the 10th cryopreservation of the same $\mathrm{UC}$ tissue with subsequent thawing and explant reculture $\left(\mathrm{MSC}_{\mathrm{UC}-\mathrm{re}(10)}\right)$ (Fig. 7a). These findings were also substantiated by flow cytometry analysis demonstrating expression of CD73, CD90, and CD105 in more than $90 \%$ of selective populations every third freeze/thaw/explant 


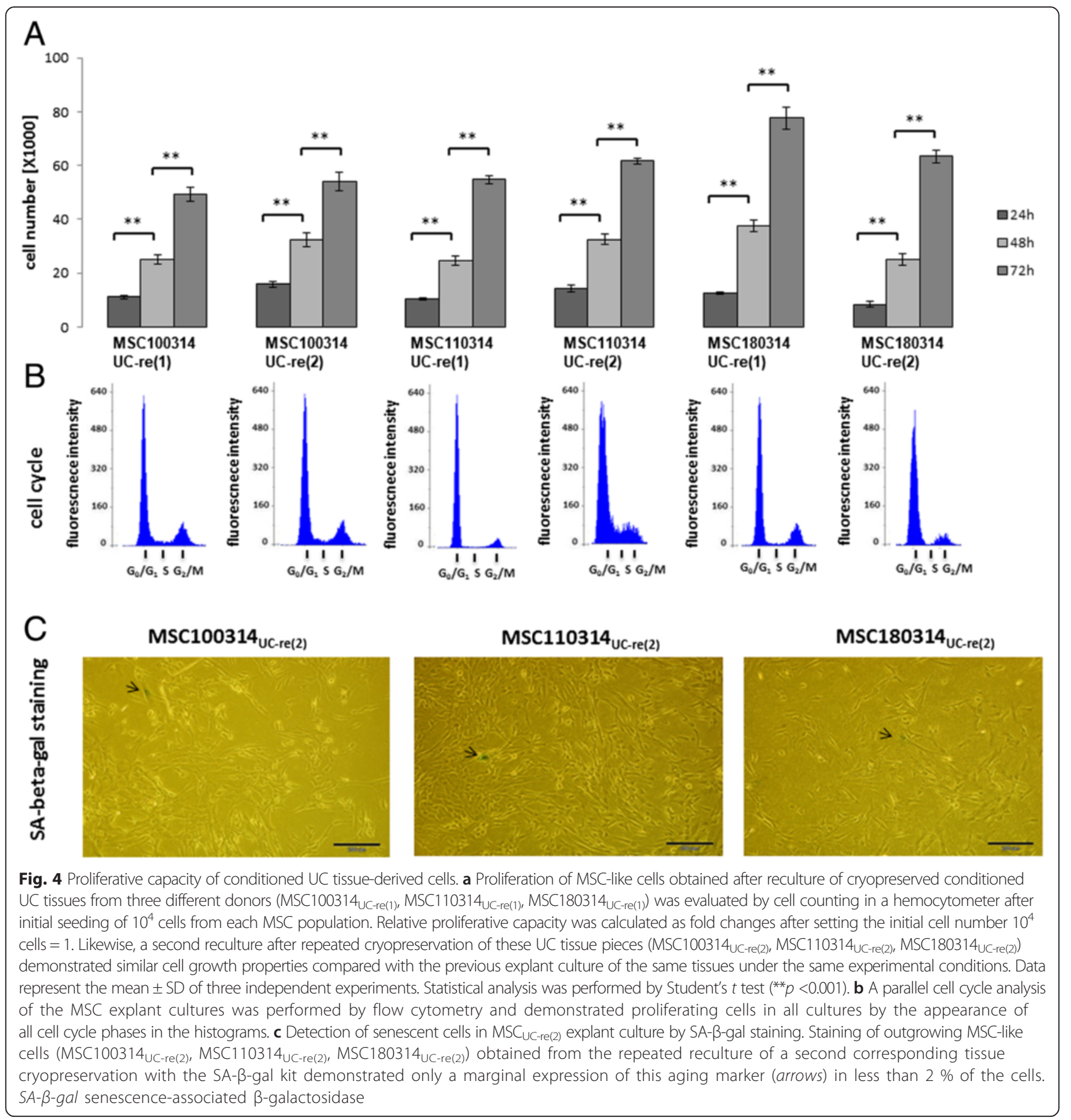

culture cycle, respectively (Fig. 7b). The proliferative capacity of these MSC from conditioned UC tissues after one, four, seven, and 10 freeze/thaw/explant culture cycles revealed little if any differences between one and four freeze/thaw/explant culture cycles; however, seven and 10 freeze/thaw/explant culture cycles were associated with a significantly reduced proliferative capacity (Fig. 7c). These findings were substantiated by determination of the MSC yield from consecutive 10-day
UC tissue explant cultures demonstrating a progressive decrease of outgrowing MSC after six freeze/thaw/explant culture cycles (Fig. 7a). The data demonstrated an accumulated yield of about $3.3 \times 10^{7}$ MSC from $5.2 \mathrm{mg}$ UC tissue within 10 days. Based on an average UC length of 50-60 cm with $1.5-2 \mathrm{~cm}$ diameter and $41 \mathrm{~g}$ weight, an optimal primary explant culture using the whole UC tissue could initially yield about $2.6 \times 10^{11}$ MSC. In the case of fresh UC tissue, conditioning 


\section{MSC explant culture after one (1) and two (2) UC tissue cryo-preservations}

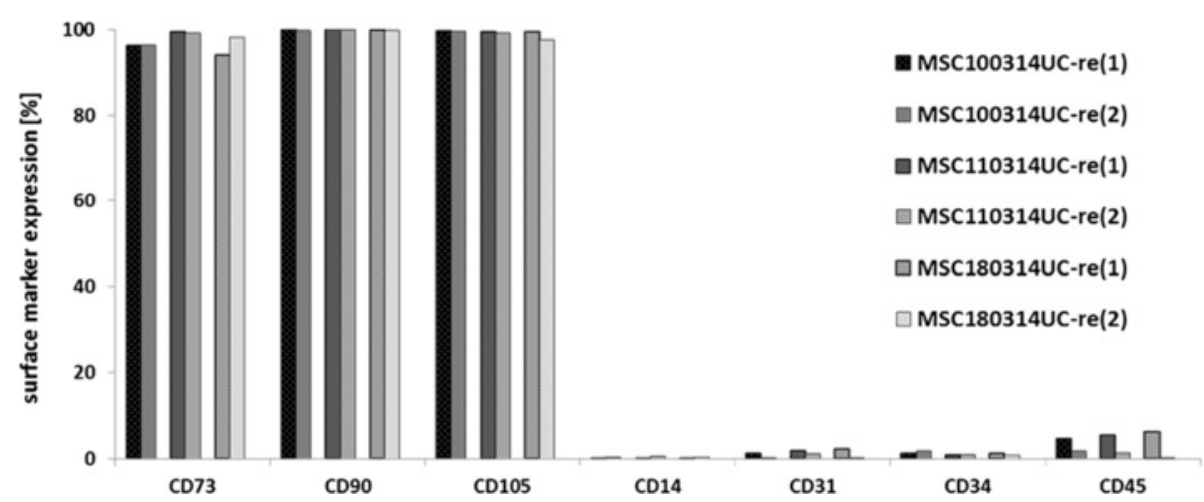

Fig. 5 MSC surface marker analysis. Cell surface marker evaluation was performed for the presence of the typical MSC markers (CD73, CD90, and CD105) by flow cytometry analysis in cells of three individual UC explant cultures of the previously cryopreserved UC tissues. a MSC100314UC-re(1). b MSC110314uc-re(1). c MSC180314uC-re(1). Moreover, the MSC marker expression was compared with cells obtained after second reculture following repeated cryopreservation of these UC tissue pieces. d MSC100314 UC-re(2). e MSC110314UC-re(2). f MSC180314UC-re(2). A simultaneous absence of further cell type-specific surface markers CD31, CD34, CD45, and CD14 was tested. The analysis was performed in steady state-growing cells by flow cytometry compared with the corresponding immunoglobulin isotype antibodies which were used as appropriate controls in the histograms. MSC mesenchymal stroma/stem cells

during explant culture would last about 2-3 weeks, and thereafter continuous explant culture of these tissue pieces from the whole UC could yield a total amount of more than $10^{10}$ MSC within 4 weeks. Moreover, these cells can be subcultured from each explant culture in various distinct passages to further potentiate the number of MSC.

During consecutive freeze/thaw/explant culture cycles, potential stem cell aging was quantified by the SA- $\beta$-gal assay. Whereas low levels of SA- $\beta$-gal-positive cells were observed in $\mathrm{MSC}_{\mathrm{UC}-\mathrm{re}(1)}$ as $2.8 \pm 1.3 \%$ and in $\mathrm{MSC}_{\mathrm{UC}-\mathrm{re}(4)}$ cultures as $3.9 \pm 0.3 \%$, these numbers progressively and significantly increased in populations of $\mathrm{MSC}_{\mathrm{UC}-\mathrm{re}(7)}$ to $7.8 \pm 3.3 \%$ and in $\mathrm{MSC}_{\mathrm{UC}-\mathrm{re}(10)}$ to $15.3 \pm 0.5 \%$ (Fig. $7 \mathrm{~d}$ ). Together, these findings suggested that the reduced proliferative capacity of MSC derived from 10 freeze/thaw/explant culture cycles correlated with significantly elevated amount of SA- $\beta$-gal-positive cells.

In order to address a potentially preferred homing of MSC to UC tissue rather than plastic, UC270815 tissue $(96.3 \pm 50.1 \mathrm{mg} /$ tissue; $n=4)$ was cocultured with MSC100314 ${ }^{\mathrm{GFP}}\left(1000\right.$ cells $\left./ \mathrm{cm}^{2}\right)$ in four separate experiments, whereby the original outgrowth of cells from these tissues had stopped. This coculture was performed on plastic in 24-well plates (Nunc) and cell growth was compared with a corresponding MSC monoculture at the same experimental conditions. After 6 days of culture, the UC tissue and the additional cells in the well and also the monoculture cells were lysed by SDS and aliquots were measured for fluorescence intensities and calculated to the appropriate amount of cells. Whereas $6.65 \pm 0.48 \times 10^{4} \mathrm{MSC}^{\mathrm{GFP}}$ were detected in the monoculture, the coculture was associated with $5.51 \pm 0.62 \times 10^{4}$ MSC $^{\mathrm{GFP}}$ attached to the plastic in the well and an additional $2.26 \times 10^{4} \mathrm{MSC}^{\mathrm{GFP}}$ were associated with the UC tissue (Fig. 8). These findings suggested enhanced cell growth in the presence of UC tissue and a preferred localization of $\mathrm{MSC}^{\mathrm{GFP}}$ in the UC tissue since the amount of MSC plastic adherence in the UC tissue coculture was markedly lower as compared with the MSC plastic adherence in the monoculture. Indeed, a fluorescence micrograph confirmed incorporation of MSC ${ }^{\mathrm{GFP}}$ into the rim of the UC tissue (Fig. 8). Additional preliminary observations were obtained following three extensive washes with PBS and further culture of the $\mathrm{MSC}^{\mathrm{GFP}}$-incorporated UC tissue $(61.6 \mathrm{mg}$ ) in a separate new well for 7 days, which was associated with a significantly enhanced proliferation reaching $1.2 \times 10^{5}$ MSC $^{\text {GFP }}$ within the tissue similar to a conditioning of the tissue. Moreover, about $10^{3}$ cells were detected as explant culture from this tissue. These preliminary findings were substantiated by a fluorescence micrograph demonstrating the whole UC tissue filled with $\mathrm{MSC}^{\mathrm{GFP}}$ and additional outgrowth of some cells (data not shown) which indicated conditioning of the UC tissue.

\section{Discussion}

Multipotent human MSC represent a preferred tool for cell or tissue replacement therapy and are involved in a large variety of clinical studies in regenerative medicine. 


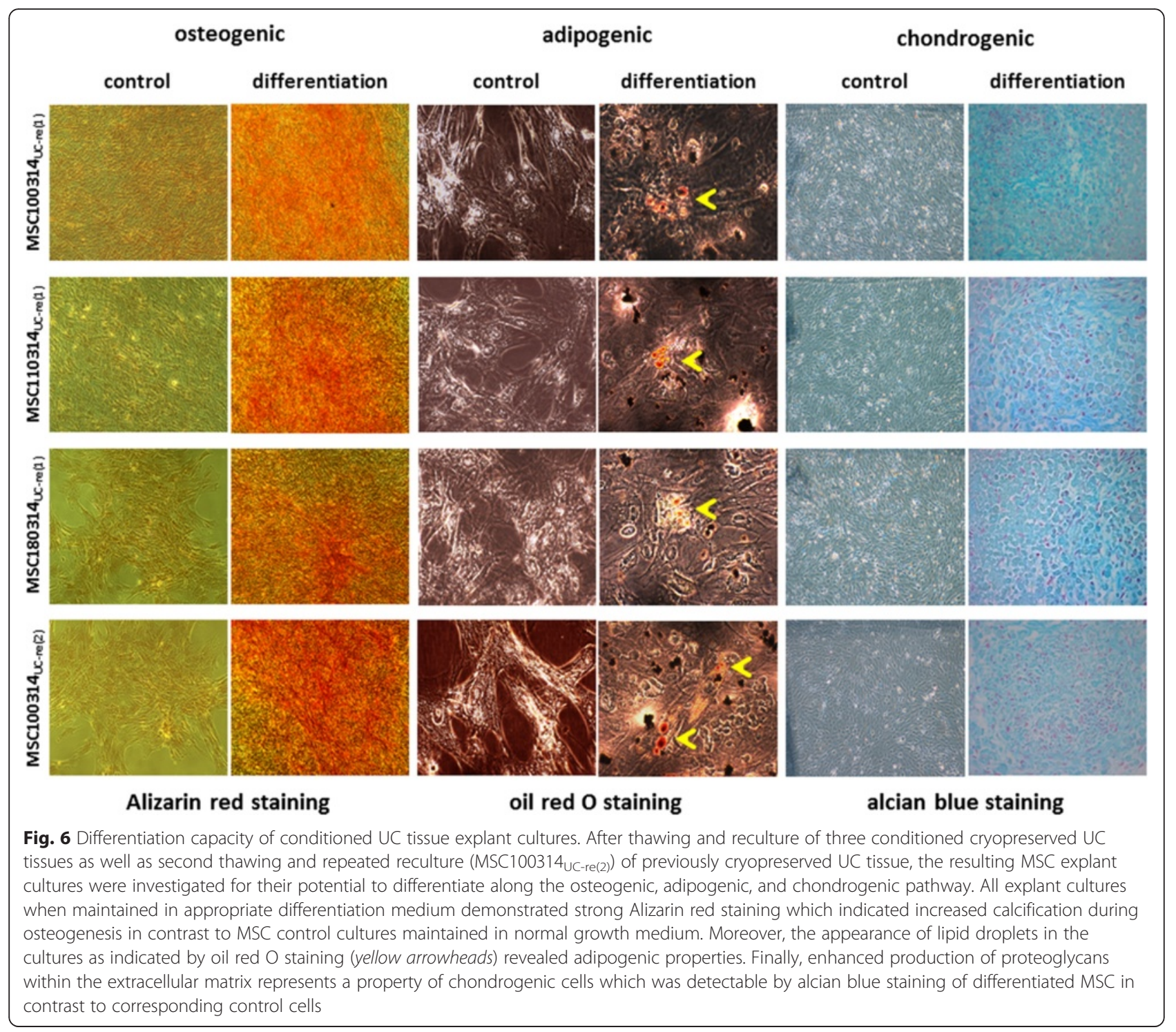

To meet the progressively increasing requirement of MSC as a more frequently used stem cell population in tissue engineering, UC provides a noninvasive, easily accessible, and ethically noncontroversial MSC-rich stem cell source [4]. Moreover, cryostorage of MSCoriginating tissue significantly elevates the availability of reproducible material and potential cryoconditions for tissues and other biospecimens have been tested previously [28]. Accordingly, cryopreservation of conditioned UC tissue in liquid nitrogen demonstrated a constant availability of MSC with reproducible properties including proliferative capacity, the maintenance of certain plasticity, and stable stem cell characteristics.

All MSC obtained after reculture of previously cryopreserved UC tissues from various donors demonstrated at least differentiation capacity along mesodermal lineages, including acquisition of osteogenic, adipogenic, and chondrogenic properties. Moreover, UC tissue and the corresponding explant cultures expressed CD73, CD90, and CD105, representing the minimal criteria for MSC markers. Previous work demonstrated that some MSC subpopulations also carry STRO-1 [29], or the receptors vascular cell adhesion molecule-1 (VCAM-1, CD106) and intercellular cell adhesion molecule-1 (ICAM-1, CD54) [30] preferably expressed in bone marrow-derived MSC. In addition, octamer-binding transcription factor-4 (OCT-4) and (sex determining region Y)-box-2 (SOX2) as embryonic-like stem cell markers can be detected in certain MSC subpopulations [31], further substantiating MSC heterogeneity and 


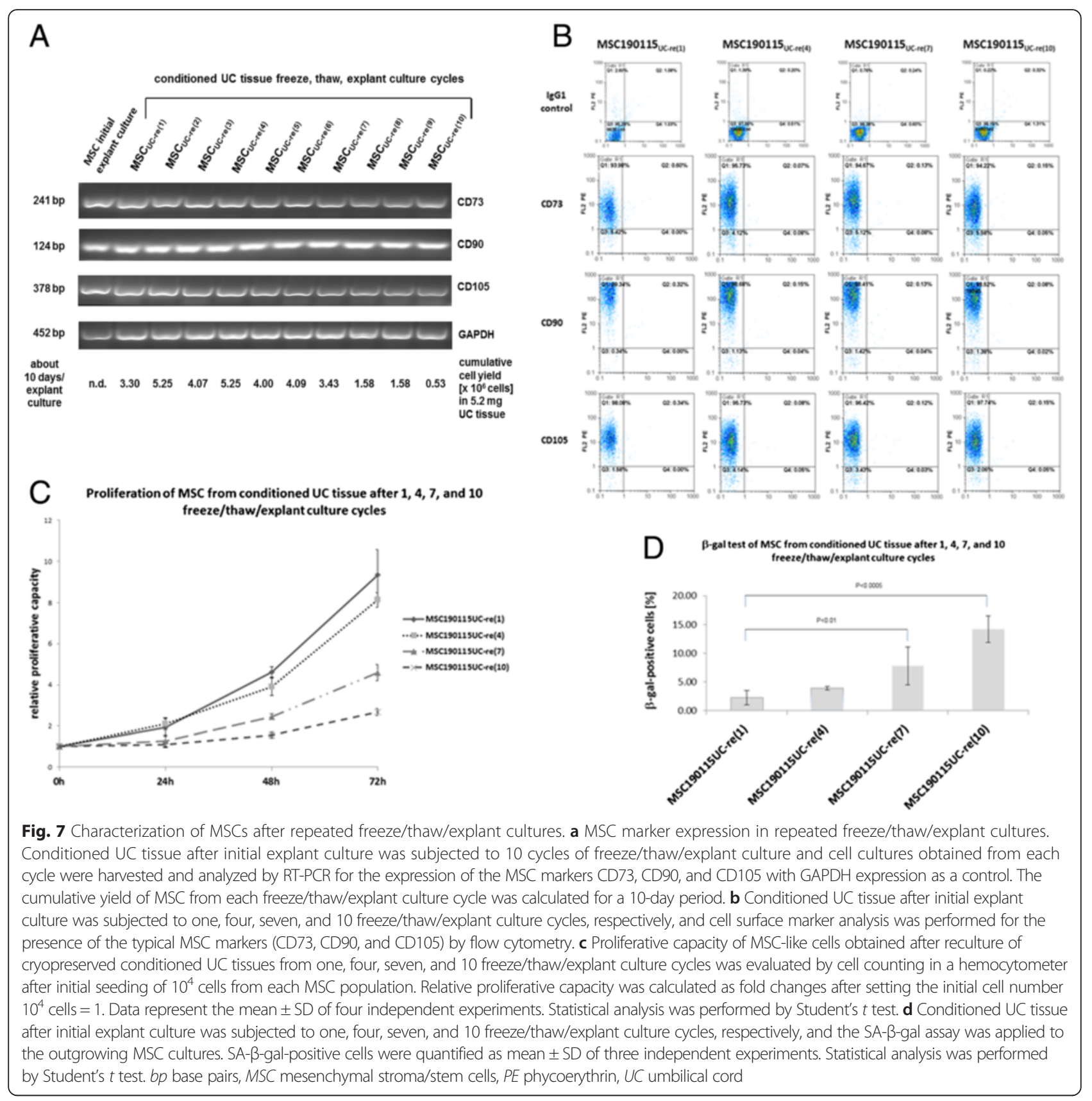

plasticity by the appearance, acquisition, or loss of distinct properties. Furthermore, MSC contribute to a transfer and exchange of markers with neighboring tissue cells, thereby altering cellular functionality by enhanced plasticity $[25,32,33]$.

Conditioning of UC tissue prior to cryopreservation by initial culture for about 2-3 weeks demonstrated proliferation of cells within the tissue to reach certain intratissue saturation. This was followed by active outgrowth of cells carrying typical MSC markers during explant culture.
Different isolation protocols to obtain MSC from UC tissue include enzymatic digestion of the extracellular matrix by either trypsin, collagenase, or accutase treatment, in contrast to another technique which is keeping an intact and physiological extracellular matrix by explant culture of MSC from UC tissue pieces. Whereas certain results favor enzymatic tissue treatment [34], other studies demonstrated an advantage of MSC explant culture [35]. However, differences in MSC culture conditions including various media and, more importantly, the use of poorly defined additives such as FCS 


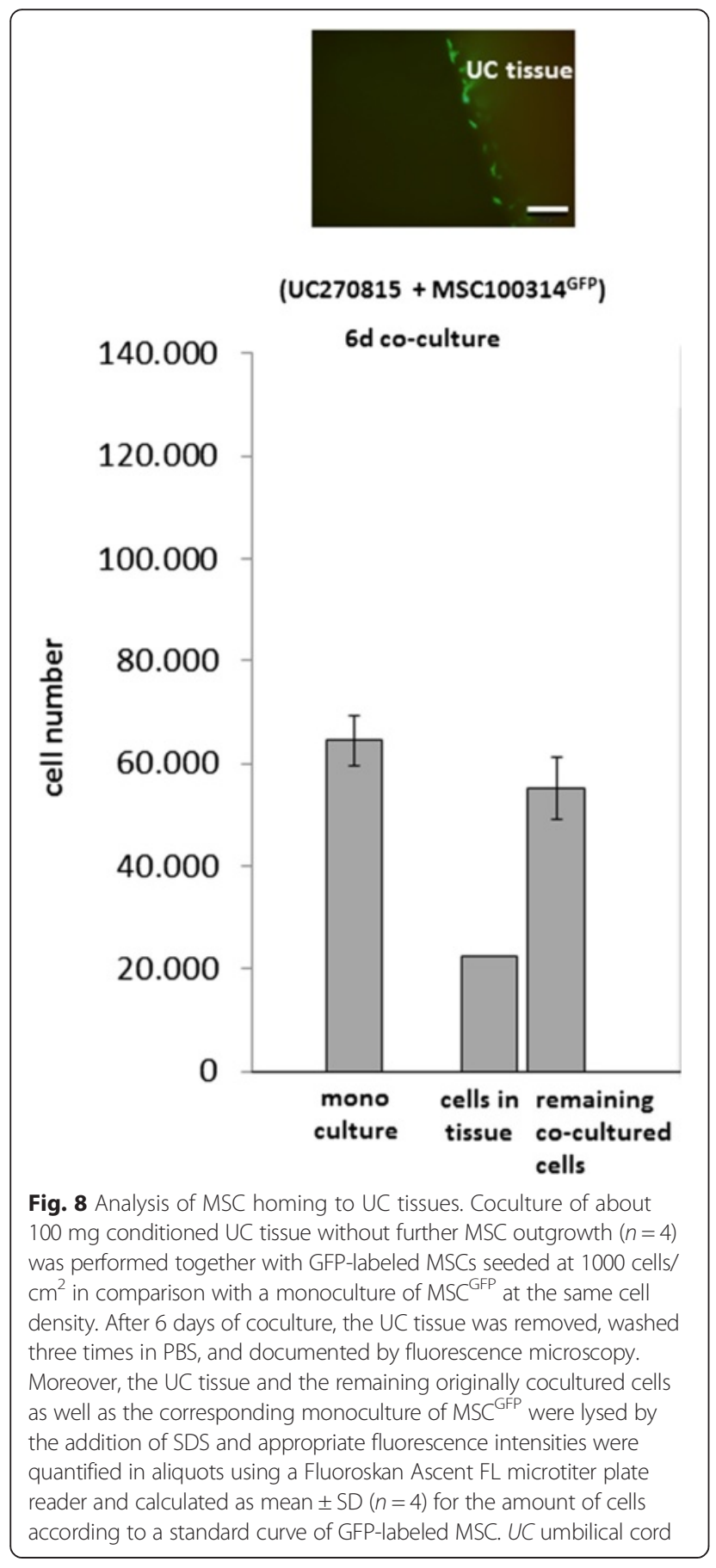

instead of xeno-free components further contribute to heterogeneity of the data.

Previous work has suggested that the enzyme-free explant culture ensures the maintenance of an intact microenvironment within the tissue pieces to keep a natural three-dimensional stroma structure for MSC with appropriate surface interaction sites [5]. Interestingly, this MSC-like outgrowth during explant culture was significantly accelerated after reculture of cryopreserved conditioned UC tissue when the UC tissue structure became more condensed by contracting forces. Other studies observed a gradient of cell maturity within the UC tissues [36] and considered the cytoskeletal complexity in UC tissue, which supports the idea of a location-dependent and microenvironment-dependent growth and differentiation potential of UC-derived MSC [36, 37].

In addition to the results of the UC tissues in this study, storage of MSC cultures can also be performed by cryopreservation in liquid nitrogen [38]. However, subculture of MSCs is associated with limited expansion until approximately 10 passages $[5,39]$. Similar findings were obtained during repeated freeze/thaw/explant culture cycles whereby reduced proliferative capacity after 10 cycles was accompanied by elevated senescence in the culture as determined by an increased amount of SA- $\beta$-gal-positive cells. In addition, previous work has demonstrated prolonged culture of MSC by keeping the originating UC tissue structure to provide a more physiological three-dimensional microenvironment for explant culture [5]. Indeed, the comparison of MSC culture on plastic versus UC tissue demonstrated a preferred homing of MSC to UC tissue.

\section{Conclusion}

In summary, these findings suggested that maintenance of MSC in a three-dimensional culture enables the establishment of a natural microenvironment providing long-term outgrowth potential of stem cell-like cells with unchanged growth properties for at least four freeze/thaw/explant culture cycles. Moreover, repeated cryopreservation of conditioned UC tissues keeps stroma/stem cell multiplicity and thereby minimizes a likely culture condition-mediated clonal selection or progressive clonal convergence towards an accumulation of certain subpopulations. Consequently, these tissues provide a microenvironment for maximal plasticity with reasonable amounts of originating MSC which is required in the field of regenerative medicine. Moreover, this more natural three-dimensional stem cell culture also enables UC tissue banking with reproducible availability of MSC for cell therapies and tissue engineering.

\footnotetext{
Abbreviations

aMEM: Minimal Essential Medium Eagle, alpha-modification; bp: Base pairs; BSA: Bovine serum albumin; DAPI: 4,6-Diamidine-2-phenylindoldihydrochloride; DMEM: Dulbecco's modified Eagle's medium; DMSO: Dimethyl sulfoxide; FBS: Fetal bovine serum; FCS: Fetal calf serum; FITC: Fluorescein isothiocyanate; GAPDH: Glyceraldehyde 3-phosphate dehydrogenase; GFP: Green fluorescent protein; HLA-DR: Human leukocyte antigen DR; HS: Human AB-serum; ICAM1: Intercellular cell adhesion molecule-1; MSC: Mesenchymal stroma/stem cells; OCT-4: Octamer-binding transcription factor-4; PBS: Phosphate-buffered saline; PE: Phycoerythrin; RT: Reverse transcription; SA- $\beta$-gal: Senescence-associated
} 
ß-galactosidase; SD: standard deviation; SOX-2: (Sex determining region Y)-box-2; UC: Umbilical cord; VCAM-1: Vascular cell adhesion molecule-1.

\section{Competing interests}

Each author declares that competing financial interests have been appropriately disclosed according to the policy of the Journal. The authors further declare no personal or professional conflict of interest, whether they are actual or potential

\section{Authors' contributions}

$Y Y, C M$, and $A O$ performed the surface marker analysis and the proliferation studies. JvdO contributed the data of the RT-PCR and $\beta$-gal assay. VB performed the differentiation studies. RH established the cryopreservation, UC explant cultures and morphology, MSC primary cells, performed the lentiviral transduction, designed the study, and drafted the manuscript. All authors critically read and approved the final manuscript.

\section{Acknowledgements}

UC tissue from previous studies and test tissue was provided by Prof. C $\mathrm{V}$ Kaisenberg, MHH women's clinic, Hannover, Germany. YY is a postdoctoral research fellow from Tongji University, Shanghai, China.

\section{Author details}

'Department of Obstetrics and Gynecology, Biochemistry and Tumor Biology Laboratory, Hannover Medical School, Carl-Neuberg-Str. 1, Hannover D-30625, Germany. ${ }^{2}$ Tongji Hospital Affiliated Tongji University, Shanghai 200065, China. ${ }^{3}$ Department of Plastic, Hand and Reconstructive Surgery, Hannover Medical School, Carl-Neuberg-Straße 1, Hannover D-30625, Germany. ${ }^{4}$ Department of Gynecology and Obstetrics, Biochemistry and Tumor Biology Laboratory, Hannover Medical School, Carl-Neuberg-Straße 1, Hannover D - 30625, Germany.

\section{Received: 4 September 2015 Revised: 21 January 2016}

\section{Accepted: 27 January 2016 Published online: 11 February 2016}

\section{References}

1. Friedenstein AJ, Gorskaja JF, Kulagina NN. Fibroblast precursors in normal and irradiated mouse hematopoietic organs. Exp Hematol. 1976;4(5):267-74.

2. Fraser JK, Wulur I, Alfonso Z, Hedrick MH. Fat tissue: an underappreciated source of stem cells for biotechnology. Trends Biotechnol. 2006;24(4):150-4.

3. Wolbank S, van Griensven M, Grillari-Voglauer R, Peterbauer-Scherb A. Alternative sources of adult stem cells: human amniotic membrane. Adv Biochem Eng Biotechnol. 2010;123:1-27

4. Hass R, Kasper C, Bohm S, Jacobs R. Different populations and sources of human mesenchymal stem cells (MSC): a comparison of adult and neonatal tissue-derived MSC. Cell Commun Signal. 2011;9:12.

5. Otte A, Bucan V, Reimers K, Hass R. Mesenchymal stem cells maintain longterm in vitro stemness during explant culture. Tissue Eng. 2013:19(12):937-48.

6. Caplan Al. Mesenchymal stem cells. J Orthop Res. 1991;9(5):641-50.

7. Majore I, Moretti P, Hass R, Kasper C. Identification of subpopulations in mesenchymal stem cell-like cultures from human umbilical cord. Cell Commun Signal. 2009;7:6.

8. Bianco P. "Mesenchymal" stem cells. Annu Rev Cell Dev Biol. 2014;30:677-704

9. Pittenger MF, Mackay AM, Beck SC, Jaiswal RK, Douglas R, Mosca JD, et al. Multilineage potential of adult human mesenchymal stem cells. Science. 1999;284(5411):143-7.

10. Dominici M, Le Blanc K, Mueller I, Slaper-Cortenbach I, Marini F, Krause D, et al. Minimal criteria for defining multipotent mesenchymal stromal cells. The International Society for Cellular Therapy position statement. Cytotherapy. 2006;8(4):315-7.

11. Wolf $K$, Alexander S, Schacht $V$, Coussens LM, von Andrian UH, van Rheenen $\mathrm{J}$, et al. Collagen-based cell migration models in vitro and in vivo. Semin Cell Dev Biol. 2009;20(8):931-41.

12. Kraus $\mathrm{KH}$, Kirker-Head $\mathrm{C}$. Mesenchymal stem cells and bone regeneration. Vet Surg. 2006;35(3):232-42.

13. Bornes TD, Adesida AB, Jomha NM. Mesenchymal stem cells in the treatment of traumatic articular cartilage defects: a comprehensive review. Arthritis Res Ther. 2014;16(5):432.

14. Horwitz EM, Prockop DJ, Gordon PL, Koo WW, Fitzpatrick LA, Neel MD, et al. Clinical responses to bone marrow transplantation in children with severe osteogenesis imperfecta. Blood. 2001;97(5):1227-31.
15. Le Blanc K, Rasmusson I, Sundberg B, Gotherstrom C, Hassan M, Uzunel M, et al. Treatment of severe acute graft-versus-host disease with third party haploidentical mesenchymal stem cells. Lancet. 2004;363(9419):1439-41.

16. Sykova $E$, Jendelova P, Urdzikova L, Lesny P, Hejcl A. Bone marrow stem cells and polymer hydrogels two strategies for spinal cord injury repair. Cell Mol Neurobiol. 2006;26(7-8):1113-29.

17. Perin EC, Dohmann HF, Borojevic R, Silva SA, Sousa AL, Silva GV, et al. Improved exercise capacity and ischemia 6 and 12 months after transendocardial injection of autologous bone marrow mononuclear cells for ischemic cardiomyopathy. Circulation. 2004;110(11 Suppl 1):I1213-8.

18. Lazarus HM, Koc ON, Devine SM, Curtin P, Maziarz RT, Holland HK, et al. Cotransplantation of HLA-identical sibling culture-expanded mesenchymal stem cells and hematopoietic stem cells in hematologic malignancy patients. Biol Blood Marrow Transplant. 2005;11(5):389-98.

19. Serikov V, Hounshell C, Larkin S, Green W, Ikeda H, Walters MC, et al. Human term placenta as a source of hematopoietic cells. Exp Biol Med (Maywood). 2009:234(7):813-23.

20. Choudhery MS, Badowski M, Muise A, Harris DT. Utility of cryopreserved umbilical cord tissue for regenerative medicine. Curr Stem Cell Res Ther. 2013;8(5):370-80.

21. Choudhery MS, Badowski M, Muise A, Pierce J, Harris DT. Cryopreservation of whole adipose tissue for future use in regenerative medicine. J Surg Res. 2014;187(1):24-35

22. Donnez J, Dolmans MM. Fertility preservation in women. Nat Rev. 2013; 9(12):735-49.

23. Bissoyi A, Nayak B, Pramanik K, Sarangi SK. Targeting cryopreservationinduced cell death: a review. Biopreserv Biobank. 2014;12(1):23-34.

24. Mandel K, Yang Y, Schambach A, Glage S, Otte A, Hass R. Mesenchymal stem cells directly interact with breast cancer cells and promote tumor cell growth in vitro and in vivo. Stem Cells Dev. 2013;22(23):3114-27.

25. Yang Y, Otte A, Hass R. Human mesenchymal stroma/stem cells exchange membrane proteins and alter functionality during interaction with different tumor cell lines. Stem Cells Dev. 2014;15;24(10):1205-22.

26. Hass R, Meinhardt G, Hadam M, Bartels H. Characterization of human TUR leukemia cells: continued cell cycle progression in the presence of phorbol ester is associated with resistance to apoptosis. Eur J Cell Biol. 1994;65(2):408-16.

27. Yegorov YE, Akimov SS, Hass R, Zelenin AV, Prudovsky IA. Endogenous betagalactosidase activity in continuously nonproliferating cells. Exp Cell Res. 1998;243(1):207-11.

28. Hubel A, Spindler R, Skubitz AP. Storage of human biospecimens: selection of the optimal storage temperature. Biopreserv Biobank. 2014;12(3):165-75.

29. Simmons PJ, Torok-Storb B. Identification of stromal cell precursors in human bone marrow by a novel monoclonal antibody, STRO-1. Blood. 1991:78(1):55-62.

30. Honczarenko M, Le Y, Swierkowski M, Ghiran I, Glodek AM, Silberstein LE. Human bone marrow stromal cells express a distinct set of biologically functional chemokine receptors. Stem Cells. 2006;24(4):1030-41.

31. Kuroda Y, Kitada M, Wakao S, Nishikawa K, Tanimura Y, Makinoshima H, et al. Unique multipotent cells in adult human mesenchymal cell populations. Proc Natl Acad Sci U S A. 2010;107(19):8639-43.

32. Ungefroren $H$, Sebens $S$, Seidl $D$, Lehnert $H$, Hass R. Interaction of tumor cells with the microenvironment. Cell Commun Signal. 2011:9:18.

33. Hass R, Otte A. Mesenchymal stem cells as all-round supporters in a normal and neoplastic microenvironment. Cell Commun Signal. 2012;10(1):26.

34. Tong CK, Vellasamy S, Tan BC, Abdullah M, Vidyadaran S, Seow HF, et al. Generation of mesenchymal stem cell from human umbilical cord tissue using a combination enzymatic and mechanical disassociation method. Cell Biol Int. 2011;35(3):221-6.

35. Priya N, Sarcar S, Majumdar AS, SundarRaj S. Explant culture: a simple, reproducible, efficient and economic technique for isolation of mesenchymal stromal cells from human adipose tissue and lipoaspirate. J Tissue Eng Regen Med. 2014;8(9):706-16.

36. Nanaev AK, Kohnen G, Milovanov AP, Domogatsky SP, Kaufmann P. Stromal differentiation and architecture of the human umbilical cord. Placenta. 1997 18(1):53-64.

37. Kobayashi K, Kubota T, Aso T. Study on myofibroblast differentiation in the stromal cells of Wharton's jelly: expression and localization of alpha-smooth muscle actin. Early Hum Dev. 1998;51(3):223-33.

38. Luetzkendorf J, Nerger K, Hering J, Moegel A, Hoffmann K, Hoefers C, et al. Cryopreservation does not alter main characteristics of Good 
Manufacturing Process-grade human multipotent mesenchymal stromal cells including immunomodulating potential and lack of malignant transformation. Cytotherapy. 2015;17(2):186-98.

39. Kulterer B, Friedl G, Jandrositz A, Sanchez-Cabo F, Prokesch A, Paar C, et al. Gene expression profiling of human mesenchymal stem cells derived from bone marrow during expansion and osteoblast differentiation. BMC Genomics. 2007;8:70.

Submit your next manuscript to BioMed Central and we will help you at every step:

- We accept pre-submission inquiries

- Our selector tool helps you to find the most relevant journal

- We provide round the clock customer support

- Convenient online submission

- Thorough peer review

- Inclusion in PubMed and all major indexing services

- Maximum visibility for your research

Submit your manuscript at www.biomedcentral.com/submit 